\title{
Divergence \& Probability Density : A Hypothesis
}

By

\section{Datta et al}

Multiplicative Probability, $a^{\wedge} x$ series , Hypothesis , Probability Density

The earlier work by Datta et al ( 1 ) the note has been made with the algebraic properties

of Probability ie $P(A)+P(B)=P\left(A^{*} B\right)$. This is termed as multiplicative rule of

Probability. By multiplication rule any number say a can be first diverged through a series of a (

pow) $x$, Where $x$ is an integer like 0 to $n$.

Discussion : Assume $\mathrm{a}=1=1{ }^{*} 1 \Rightarrow 1+1=2$

$$
\begin{aligned}
& \Rightarrow 22^{*} 2=4 \text { ( Multiplicative ) }=2^{\wedge} 2 \\
& \Rightarrow 4^{\star} 4=16=2^{\wedge} 4 \\
& \Rightarrow 16^{*} 16=256=2^{\wedge} 8 \\
& \Rightarrow 256^{\star} 256=65536=2^{\wedge} 16 \\
& \Rightarrow 65536{ }^{\star} 65536=\text { The result is a Divergence Series } \\
& \Rightarrow \text { le Now adding all the result of } P(A)+P(B)=P\left(A^{\star} B\right) \\
& \Rightarrow 1+a^{\wedge} x=\text { where } a=1 \& x=32
\end{aligned}
$$

So $P\left(A^{*} B\right)$ function $=42$. .10 digit $=>$ Taking $\ln$ in both side we get

$$
\begin{gathered}
\ln P\left(A^{*} B\right)=\ln \left(2^{\wedge} 32\right)=32 * 0.69314 \ldots . .13 \text { digit } \\
\text { Assuming } 5 \text { digit }=>\text { Taking } \exp (22.18048)=4293 \ldots \ldots . .6 \text { digit } .1657
\end{gathered}
$$

This may be Considered as Hypothesis or may not be !!

Conclusion : The above simplest hypothesis will explain the Probability to Probability density $f(x)$ ie $P\left(A^{*} B\right)$. Which is a good numerical fit with our earlier work.

REF :

1. JAYDIP DATTA et al, A brief trip to the Universe of Numbers. Mathematical Disclosure Series , March 2020 ,DOI: 10.13140/RG.2.2.24323.66082

(https://www.researchgate.net/publication/339901086 A brief trip to the Universe of Numb ers Mathematical Disclosure Series? sg=0SI1Z9pJP4JwXU5wrBJv-1CRUf5VIPFLg1LLRD33GCbGa0I ojFZaWmpIDKJSwSBF--Lj-MUyoish5eZUatJ1Y5NtJKFN4Sck15feS.uekuaHehHM-IJX-zJ8 5MkRQasHAMQQHuboWGBAuy-Q 8lo9gSlyGQFh4DCDkyw6jyXa-x9MButvyT5t2iZig ) 
2. Datta et al ( 1 ), PROBABILITY \& WAVEFUNCTION : A NOTE 
